\title{
Corela
}

Cognition, représentation, langage

HS-31 | 2020

Métalinguistiques.

\section{Énoncés métalangagiers en contraste :}

Activités métalinguistiques dans différents genres du discours puriste français

Vera Neusius

\section{(2) OpenEdition}

\section{Journals}

Édition électronique

URL : http://journals.openedition.org/corela/11636

DOI : 10.4000/corela.11636

ISSN : 1638-573X

Éditeur

Cercle linguistique du Centre et de l'Ouest - CerLICO

\section{Référence électronique}

Vera Neusius, «Énoncés métalangagiers en contraste : », Corela [En ligne], HS-31 | 2020, mis en ligne le 02 juillet 2020, consulté le 03 juillet 2020. URL : http://journals.openedition.org/corela/11636 ; DOI : https://doi.org/10.4000/corela.11636

Ce document a été généré automatiquement le 3 juillet 2020

\section{(c) (i) (2)(2)}

Corela - cognition, représentation, langage est mis à disposition selon les termes de la licence Creative Commons Attribution - Pas d'Utilisation Commerciale - Partage dans les Mêmes Conditions 4.0 International. 


\section{Énoncés métalangagiers en contraste :}

Activités métalinguistiques dans différents genres du discours puriste français

Vera Neusius

\section{Remarques préliminaires}

1 En France, le discours sur la langue constitue depuis le 15 e siècle ${ }^{1}$ un "fait social " (Schmitt 2000: 682 ; Polzin-Haumann 2008: 1472). Ce discours métalinguistique, parfois sous la forme d'interventions normatives plus ou moins conscientes sur la langue, son usage ou le comportement linguistique de ses locuteurs, parfois sous celle de commentaires sur la situation linguistique en général, fait l'objet d'une longue tradition de recherche en linguistique (Eckkrammer \& Leascure 2015: 133). Ces interventions sur la langue, ou sur les langues, ayant pour objectif d'influencer, de déterminer et de normaliser le comportement linguistique s'inscrivent - à côté d'autres sphères d'activités officielles et informelles - dans le domaine de l'aménagement linguistique (Eckkrammer \& Leascure 2015: 134 ; Labrie \& Nelde 1994 ; Polzin-Haumann 2008). Cette volonté de défendre et de promouvoir la langue française a pris naissance au $16^{\mathrm{e}}$ siècle, notamment en 1549 avec la publication de Deffense et illustration de la langue francoyse de Joachim du Bellay; cette même idée s'est dès lors inscrite dans une tradition de réflexions métalinguistiques et «d'interventionnisme linguistique » (Rosier 2004: 69), qui peut être décrite en première approximation comme "discours normatif " (Berrendonner 1982).

2 À partir des années 1960 les recherches sociolinguistiques suggéraient d'envisager la situation linguistique non seulement dans le cadre législatif, mais aussi dans sa dimension d'interactions sociales, d'où, selon les différentes idéologies, l'apparition, dans la terminologie scientifique, de notions comme planification linguistique (Haugen 1959, "language planning"), ingénierie linguistique, normalisation linguistique (Teulat 1975) 
ou le terme rarement utilisé de glottopolitique (Guespin \& Marcellesi 1968; PolzinHaumann 2008: 1473).

3 Pour les réflexions qui suivront, nous prendrons pour point de départ la notion de planification linguistique que Cooper (1989: 45) définit ainsi : «Language planning refers to deliberate efforts to influence the behaviour of others with respect to the acquisition, structure, or functional allocation of their language codes ». Selon cette définition, même les réflexions métalinguistiques situées hors du cadre relativement restreint de la planification officielle et de la linguistique, présentent un intérêt scientifique.

Ces interventions, dites 'populaires', sur la question de la langue font depuis le nouveau millénaire l'objet de recherches sociolinguistiques dans le cadre de la linguistique appliquée (Osthus 2015: 160 ; Wilton \& Stegu 2011). Ce domaine tire son origine du programme fondateur de la folk linguistics de Hoenigswald, qui le définit par la proposition suivante, traduite par Beacco (2004a: 3) : «On devrait s'intéresser non seulement à (a) ce qui se passe (le langage) mais aussi (b) à la manière dont les gens réagissent à ce qui se passe [...] et aussi (c) à ce que les gens disent à propos de ce qui se passe (les propos relatifs au langage)». Dès lors, la linguistique populaire, champ d'études et discipline en même temps, s'est imposée à l'échelle internationale, à partir des États-Unis, surtout avec l'ouvrage publié en 1999 par Preston \& Niedzielski, lequel fournit un aperçu significatif des études dédiées à « découvrir les faits de la langue à travers des conceptualisations populaires » (Osthus 2015: 161).

5 En sciences du langage, la description des discours métalinguistiques des non-experts se manifeste dans plusieurs domaines de recherche comme la didactique des langues, les recherches sur le discours normatif ou la dialectologie (ibid.), bien que son instabilité terminologique soit accompagnée d'une représentation encore relativement marginale de la discipline dans le champ scientifique. Ainsi, en opposition à linguistique populaire, la philologie romane, par exemple, «a créé le terme linguistique des profanes, probablement en analogie à l'allemand [...]» (ibid.), le terme Laien-Linguistik ayant été proposé en 1996 par Gerd Antos. La variété des désignations, "[...] par tout autre adjectif stabilisé ${ }^{2}$ dans le domaine comme profane, spontané, sauvage, naïf, laien ou lay », témoigne d'une instabilité de dénomination issue d'un champ d'investigation particulièrement riche (Paveau 2008: 94).

\section{Métalangage et métadiscours}

L'ouvrage de Rey-Debove sur le métalangage naturel, paru en 1978, part de la distinction entre les concepts de métalangage appliqué à la description linguistique et de métalangage appliqué à un langage formalisé (cf. Julia 2001: 29; Rey-Debove 1978). En établissant tout d'abord le principe que «chaque langue dispose des moyens de parler d'elle-même ou d'autres langues ", l'étude linguistique du métalangage naturel peut s'inscrire dans des perspectives multiples (Rey-Debove 1978; Authier-Revuz 2002: 2). Dans ce cadre, notre analyse partira d'abord du concept de métalangage naturel qui "peut se vouloir scientifique » et qui par exemple, selon Rey-Debove, "est représenté dans le discours des linguistes " (ibid.). Considérant le discours sur la langue, il reste à souligner que les descriptions métalinguistiques d'origine non scientifiques peuvent également se vouloir scientifiques ce qui renforce encore la nature hétérogène et opaque du discours métalinguistique. Pour cette raison, la question abordée dans cette 
contribution tient compte de la différence entre différents modes métalinguistiques coexistant dans le discours sur la langue, qui peuvent se manifester dans une orientation scientifique-didactique ou dans du langage courant (cf. Rey-Debove 1997: 22) ainsi que dans un mode puriste, qu'il soit expert ou non-savant. En même temps il faut souligner qu'il n'existe pas une dichotomie absolue entre ces modalités métalinguistiques, tout comme «les frontières entre purisme et linguistique ne sont pas étanches » (Paveau \& Rosier 2008: 51). Aussi le savoir d'expert et le savoir populaire sont-ils plutôt à considérer comme des pôles opposés sur un continuum (Stegu 2008: 83) :

L'idée d'une ligne de démarcation nette séparant le «scientifique » du " populaire » me semble être également une construction - ou illusion - normative, soit dans le domaine des critères internes (pensons à des œuvres didactiques et «popularisantes »), soit quant aux critères externes : la différence entre spécialistes et non-spécialistes est graduelle [...]. Un professeur de linguistique - et supposons de plus, un syntacticien - prenant position, dans une discussion à la télévision, pour ou contre les anglicismes - représente-t-il dans ce cas la linguistique officielle ou populaire? Un enseignant de langue(s) dans un lycée est-il un spécialiste en linguistique, un non-spécialiste ou bien se trouve-t-il entre les deux ?

Aussi, un examen des descriptions spontanées répondant au concept d'épilinguistique de Culioli (1987: 41; Julia 2001: 20f.) nous semble-t-il utile dans le contexte du discours sur la langue :

On peut se placer du point de vue du sujet-énonciateur-locuteur qui a une activité métalinguistique non consciente (je parle alors d'activité épilinguistique) ou qui, par des jeux de langage de tous ordres, s'adonne à la jouissance du métalinguistique. A son propos, on pourrait soutenir qu'il y a du métalinguistique, mais pas de métalangage, au sens d'un langage extérieur à la langue objet.

Suivant cette définition nous supposons qu'il existe un rapport étroit entre le comportement métalinguistique et le concept d'épilinguistique lié à ce comportement, qui s'exprime dans des activités métalinguistiques véhiculées dans le discours. Cette relation résulte de la construction plus ou moins consciente de représentations, par exemple puristes, à travers des processus cognitifs - influencés et déterminés euxmêmes par une certaine idéologie dominante, " [...] instruits dans le même dogme et dotés des mêmes schèmes de pensée et d'actions, des mêmes dispositions éthiques et politiques [...]» (Bourdieu \& Boltanski 2008: 11) - qui sont observables au niveau de l'analyse d'énoncés (cf. Julia 2001: 22).

\section{Activités métalinguistiques puristes en France}

Dans ce paragraphe, nous ne nous proposons pas de donner un aperçu complet de l'histoire du purisme, qui, souvent qualifié comme «[...] un trait typiquement français ou à tout le moins francophone ", est un phénomène qui paraît dans toutes les langues normalisées et qui peut être défini très généralement comme « une attitude normative face aux emplois considérés comme déviants ou non légitimes en regard d'une langue «homogène ", emplois que la linguistique appelle variation» (Paveau \& Rosier 2008: 36-37). Néanmoins, le discours puriste français, né au $17^{\mathrm{e}}$ siècle «[...] lorsque le français devient une langue nationale " (ibid.), se révèle dès lors composante essentielle de l'histoire de la langue française, animée par des facteurs internes et externes de l'histoire de la langue, qui s'élabore dans l'aménagement linguistique officiel autant 
qu'informel, et qui s'exprime, en premier ressort, dans la standardisation et dans la normalisation du français.

10 Une définition plus précise du purisme français comme "pratique métalinguistique ", comme le proposent Paveau \& Rosier (2008: 51), dépend de facteurs et de questions divers : qui sont les acteurs puristes (experts, non-savants, passionnés ou amoureux de la langue, élitistes, etc.) ? dans quels lieux, par quels modes de communication et dans quels genres s'expriment les puristes ? à quels domaines sociaux s'adressent-ils? Par contre, l'effort d'identifier le discours puriste sur la base de critères généralisés pourrait s'avérer néfaste, dans la mesure où, selon les mêmes auteures, force est de constater que le purisme se construit sur la base de facteurs aussi bien idéologiques que sociaux :

Le discours puriste ne relève pas d'une sphère sociale et discursive précise en raison des lieux et de la diversité des énonciateurs qui le partagent, il relève d'avantage nous semble-t-il d'un ensemble de paroles et d'écrits dont le point commun serait celui du "discours tenu sur la langue ", ensemble qui se reconfigure, se réénonce, selon les lieux où il circule. Les idéologies sous-jacentes portées par le purisme [...] dépassent le strict rapport au langage [...] (ibid.).

Dans ce contexte, il nous semble d'autant plus important que, de nos jours, les puristes traitent « des aspects pratiques de norme et d'usage linguistique » (Osthus 2015: 164) en profitant en même temps du potentiel interactif des nouvelles technologies de l'information. L'étude de ces pratiques puristes, de plus en plus destinées à un grand public, ne peut se faire aujourd'hui sans prendre en considération le cadre interactif du réseau Internet (Osthus 2005 ; Osthus 2015: 166). Les débats virtuels constituent en effet une nouvelle plateforme d'activités métalinguistiques regroupant des préoccupations linguistiques de toute sorte comme des questions normatives sur la grammaire et l'orthographe, des questions esthétiques, des discussions autour des langues régionales, de la politique linguistique et surtout de la promotion de la langue française et de la défense de celle-ci contre les anglicismes et autres emprunts à l'anglais, ces dernières formant un axe essentiel du discours.

Dans ce contexte, notre interrogation ne vise pas à savoir si le discours puriste sur la langue produit des normes descriptives ou prescriptives et il ne s'agit pas non plus, comme le réclame par exemple Paveau, d'évaluer «la valeur des perceptions dans l'élaboration d'un discours linguistique populaire, et par conséquent la validité des théories populaires » (2007: 93). Dans notre analyse nous nous intéressons, d'un point de vue linguistique, davantage à la comparaison de la construction verbale et topique d'un journal et d'un forum sur Internet, qui - tous les deux dédiés à la promotion linguistique - constituent deux genres similaires, mais autonomes dans le discours puriste sur la langue française.

\section{Purisme et lieux d'énonciation : la question de généricité}

13 En analyse du discours, la catégorie du genre discursif est à définir « à partir de critères situationnels " (Maingueneau 2007: 30) tels que les paramètres mentionnés déjà auparavant lors de la catégorisation du discours puriste (acteurs/auteurs, medium, finalité, cadre spatiotemporel, etc.). En examinant maintenant l'ensemble des énoncés puristes - étant en même temps conscients de la complexité de cet objet de recherche 
et du fait que «le purisme pourrait sommeiller dans toute tenue du discours sur la langue » (Paveau \& Rosier 2008: 5) - nous tentons de déployer le discours puriste en fonction des genres du discours et des modes de généricité en posant la question de savoir si l'attitude puriste et la 'figure classique du puriste' varient selon l'environnement où elles s'expriment.

Nous constatons d'abord qu'une grande partie des activités métalinguistiques dans le discours puriste se transmet par des " genres institués auctoriaux » (p. ex. grammaires, dictionnaires, documents historiques, libres de style) et « routiniers» (p. ex. journaux, magazines, communiqués). En ce qui concerne les " genres routiniers", il faut souligner que :

[...] ce sont ceux qu'étudient avec prédilection les analystes du discours: le magazine, l'interview radiophonique, la dissertation littéraire [...], etc. Les rôles joués par leurs partenaires sont fixés à priori par des institutions et restent normalement inchangés pendant l'acte de communication. Ce sont ceux qui correspondent le mieux à la définition du genre du discours comme dispositif de communication défini socio-historiquement. [...] Les paramètres qui les constituent résultent en effet de la stabilisation, sous forme de normes, de contraintes liées à une activité verbale qui s'exerce dans une situation sociale déterminée. (Maingueneau 2007: 30)

Ainsi, nous proposons de classer par exemple les bulletins d'associations puristes comme Avenir de la langue française, agréé par le ministère de la Justice et déclaré au Journal officiel (voir plus bas), dans le genre institué routinier dont la scène d'énonciation est caractérisée souvent «[...] par des formules et des schèmes compositionnels préétablis sur lesquels s'exerce un fort contrôle, pour lesquels les participants sont pratiquement interchangeables. » (ibid.: 32)

Mais qu'en est-il avec des activités métalinguistiques puristes qui s'imposent dans les nouvelles textualités et par la communication multimodale sur Internet, contestant les approches de l'analyse du discours reposant sur la dichotomie oral-écrit? (Maingueneau 2014). Même si de nombreuses pratiques de communication électronique comme p. ex. les forums de discussion (voir plus bas) ressemblent à des pratiques hors $\mathrm{du}$ Web, la conception de la discursivité et des genres du discours doit quand même être repensée: "Ces évolutions [à savoir la multimodalité et la multiplication des sources] ont pour effet de modifier le regard que le chercheur porte sur les corpus, qui sont de moins en moins intégralement verbaux. » (ibid.: 177).

De surcroît - comme Beacco (2004b) l'a mis en évidence pour la linguistique populaire, qui joue un rôle important dans l'analyse du discours puriste - la catégorisation d'énonciations puristes comme un genre ou des genres discursif(s) à part relève d'une double perspective théorique : d'une part, la linguistique populaire (et par conséquent le discours métalinguistique puriste) représenterait un genre discursif en tant que domaine scientifique de représentations métalinguistiques ordinaires (puristes); d'autre part, elle le serait également en tant que «forme structurant la communication sociale, constitutive de lieux, dont la configuration relève de la conjoncture sociohistorique, dans lesquels s'ancrent les formations discursives et s'appréhende le sens sociétal. » (ibid.: 109)

Outre ces réflexions sur 'l'intérieur linguistique' du discours puriste, notre analyse se rattache aussi au mode social d'approche du discours qui donne à quelques types de discours le statut de "discours constituant" (Maingueneau \& Cossutta 1995). Selon Maingueneau et Cossutta les discours constituants - parmi lesquels ils comptent «les 
discours religieux, scientifique, philosophique, littéraire [et] juridique » (1995: 113) entraînent dans un processus performatif la formation symbolique d'une société dans l'intention de donner un sens particulier aux actes de la collectivité et de «délimiter [...] le lieu commun de la collectivité, l'espace qui englobe l'infinité des « lieux communs » qui y circulent » (ibid.). Le discours puriste, par sa finalité de construire et de défendre l'identité nationale collective en utilisant des schèmes d'argumentation (topoii) et des procédés idéologiques consolidés d'une mentalité métalinguistique formée progressivement jusqu'à nos jours, relève bien, à notre avis, des discours constituants, participant à "l'établissement d'un discours qui serve de norme et de garant aux comportements d'une collectivité. » (ibid.)

Une telle approche qui décrit le discours comme un registre symbolique, construit et organisé par la reprise de mentalités, de motifs idéologiques et historiques prônés par un certain groupe social ${ }^{3}$, remonte aux théories de Michel Foucault et surtout à l'archéologie foucaldienne définissant le discours comme un "ensemble d'énoncés en tant qu'ils relèvent de la même formation discursive " (Foucault 1969: 153). Dans cette optique, l'analyse suivante cherche à déterminer le caractère constituant du discours puriste en examinant son 'fonctionnement énonciatif' ainsi que la formation discursive de la mentalité et les attitudes puristes dans des lieux et des genres discursifs différents.

\section{5. Étude empirique}

Cette étude de cas se compose, d'un côté, de passages extraits d'articles du bulletin trimestriel de l'association Avenir de la langue française (ALF), « [...] qui rassemble tous ceux qui refusent de se soumettre à la domination absolue de la 'langue dollar' et qui veulent continuer à vivre en français en France, dans les pays francophones, dans une Europe plurilingue, dans un monde riche de toutes ses langues et cultures » (ALF 2015) et, de l'autre côté, de contributions tirées du forum de discussion $A B C$ de la langue française $(\mathrm{ABC})$, qualifié ici comme genre discursif particulier par sa scénographie numérique. L'association ALF, créée en 1992, et son bulletin sont dédiés essentiellement à la défense de la langue française, surtout contre l'influence de l'anglais et de la culture anglo-saxonne. Par contre, $A B C$ de la langue française met à disposition tout un " inventaire des ressources disponibles sur internet concernant la langue française " ( $A B C$ 2015), y compris des forums divers qui permettent d'échanger sur différents sujets liés à la langue française, comme p.ex. la promotion linguistique.

21 La question que l'on se posera au vu des réflexions théoriques précédentes est celle de savoir si des activités métalinguistiques puristes se manifestent de manière verbale et topique différente quand elles sont réalisées dans des lieux et dans des genres discursifs différents. En même temps, la comparaison des deux genres discursifs - dont un plutôt institutionnalisé, l'autre plutôt informel - nous servira de méthode pour mettre en évidence la difficulté d'identifier le discours savant et le discours populaire sur la foi de modes de communication divers.

$\mathrm{Au}$ niveau linguistique, l'étude s'intéressera prioritairement à la structure argumentative dans sa fonction de topos, concept ambigu dans le sens où il peut être adapté à des théories particulières selon des terrains de recherche différents. Pour une approche sociolinguistique nous proposons la définition de topos comme lieu commun répétitif en «[...] mettant plus largement en avant sa composante idéologique » (Rosier 
2004 ; Wengeler 2003). Ne pouvant ici regarder plus concrètement la structure syntaxique et logique des topoi au niveau des arguments concrets - comme le proposent des recherches et des modèles divers (cf. Kienpointner 1983 ; Kopperschmidt 1989 ; Ottmers 2000 ; Perelman \& Olbrechts-Tyteca 2004) - il s'agira plutôt, dans le cas du discours puriste, d'esquisser l'aperçu d'une typologie de topoï allant dans le sens de l'analyse du discours allemande (Spitzmüller 2005 ; Wengeler 2003), qui comprend topoï souvent comme catégories ou prémisses de contenu faites sur le même moule et parfois fortement stéréotypisées (Wengeler 2003: 256, «Oberprämissen einer Argumentation »).

En examinant d'abord l'intégralité des articles du bulletin ALF, nous pouvons observer pour l'année 2011, qui nous servira d'exemple, un discours métalinguistique qui se réfère à plusieurs catégories topiques, déterminées par une analyse de texte qualitative, comme le montre ce tableau :

Tableau Catégories topiques déterminés pour le bulletin Avenir de la langue française (l'année 2011)

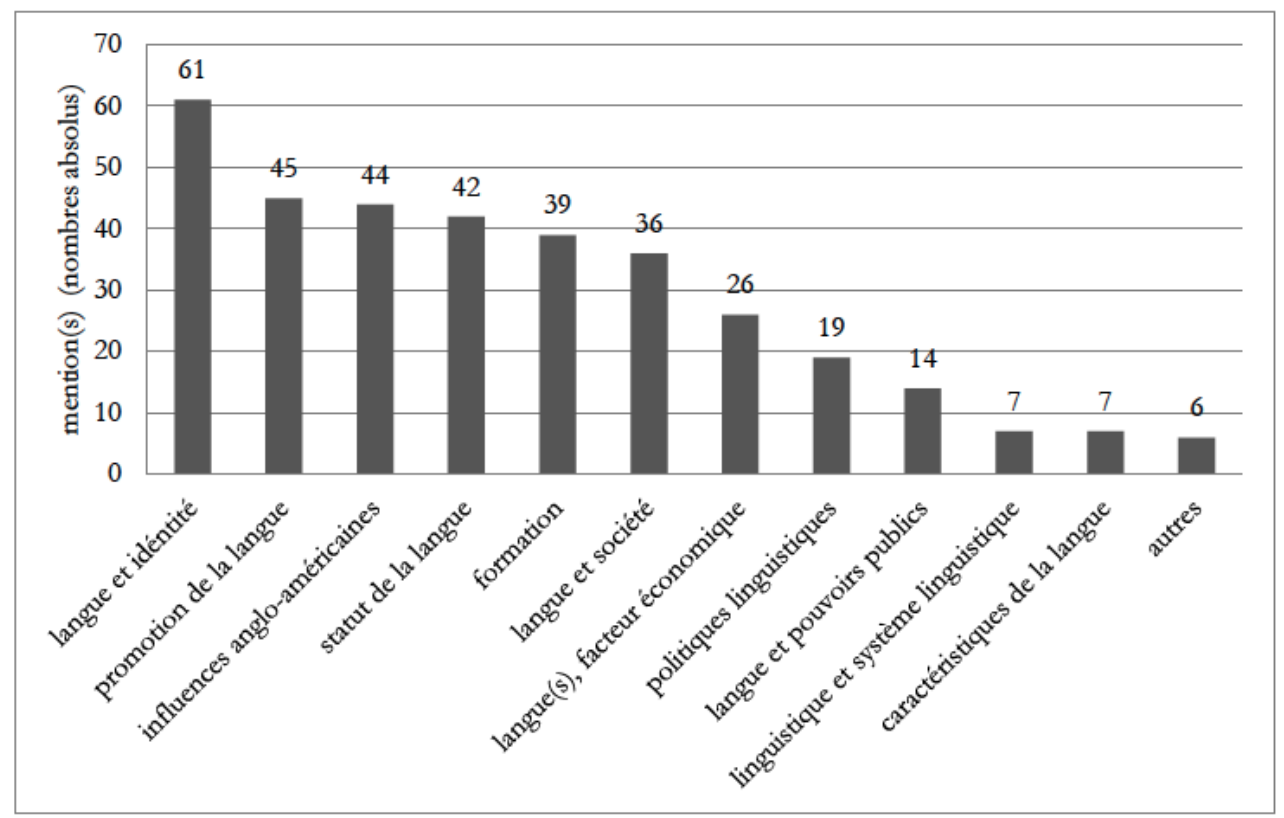

Les chiffres montrent, dans un premier temps, la fréquence des topoï mentionnés dans le bulletin, parmi lesquels 'langue et identité,, 'promotion linguistique' et 'formation' (scolaire et universitaire) se détachent d'autres catégories structurant le discours. Dans un deuxième temps, un second déchiffrage topique à l'intérieur de ces catégories principales peut nous mener à une autre division en sous-catégories - illustrées dans le tableau suivant pour le topos 'langue et identité'- qui se présentent dans la plupart des cas comme des interrelations topiques. 
Tableau : Sous-catégories du topos 'langue et identité' (bulletin ALF, année 2011)

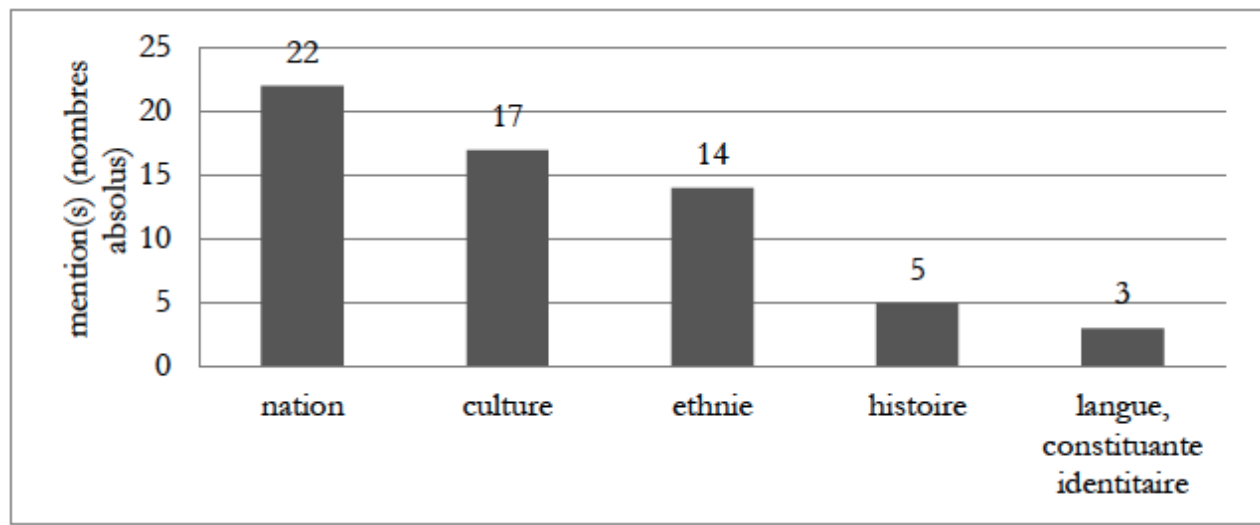
deux genres discursifs - c'est-à-dire les textes du bulletin formalisés aux énoncés métalinguistiques plus ou moins spontanés de personnes privées dans le forum de discussion - nous pouvons déterminer des structures linguistiques approximativement concordantes, notamment grâce à une référence récurrente à la Constitution française - souvent en mettant en valeur le premier alinéa «La langue de la République est le français » - et aux valeurs républicaines comme le révèlent les exemples suivants:

(1) Mais c'est le français qui est la langue de la République, et c'est le français qui est la langue de la liberté, sans compter que la France a des responsabilités qui lui sont propres au sein du monde de la francophonie. (ALF 2011-01: 12) ${ }^{4}$

(2) Si l'on s'en remet à la constitution, la langue française est la seule langue de la république en même temps qu'un élément constitutif essentiel de la nation. $(\mathrm{ABC} /$ promotion linguistique 05.08.2008)

À côté de ce lieu commun, le topos de la 'formation' se présente comme autre élément constitutif fortement véhiculé dans le bulletin ainsi que dans le réseau interactif, le plus souvent en interrelation argumentative avec la catégorie 'influences angloaméricaines' qui s'impose comme fil rouge dans chaque chaîne argumentative. Au-delà, c'est souvent le procédé métaphorique cognitif de la maladie ((3) « psychovirus » ou (4) « anglomanie ») ou le motif de la collaboration avec l'ennemi (cf. (4)) qui soutiennent la structure d'argumentation au niveau intratextuel :

(3) Charles Durand, universitaire, ancien directeur de l'Institut francophone d'informatique de Hanoï, nous rappelle que les élites des grands États européens et les officines de l'Empire anglo-saxon agissent ensemble consciemment pour détruire l'intelligence - en commençant par les langues de culture - pour mieux soumettre nos peuples à la "globalization», et les coloniser. Répandre des «psychovirus», depuis des décennies, fait partie de leur entreprise. (ALF 2011-01: 10)

(4) Il faut frapper là ou [sic] ça fait mal. Il faut décourager le suivisme abject des mercantilistes (français et étrangers) dont l'anglomanie "linguistique" commence à faire collabo, surtout quand ces organismes privés se permettent d'envahir l'espace public (affiches, abribus, téloche etc) pour le dégrader. (ABC/Promotion linguistique 04.07.2008) 
Les motifs métaphoriques s'étendent souvent sur d'autres conceptions figuratives comme la dichotomie célèbre guerre / défense (« lutte », " militants », " désertion », "défaitisme »), donnée dans les exemples (5) à (7), lesquels s'inscrivent dans le topos générique de la 'promotion linguistique' et nous permettent de constater qu'il s'agit de nouveau de la même organisation argumentative dans les deux genres discursifs.

(5) Ce texte de notre membre militant Denis Griesmar, ancien vice-président de la Société française des traducteurs, éminent linguiste et brillant essayiste, est une mine dans laquelle tous nos militants sont invités à puiser des faits, des arguments, des raisonnements dans la lutte pour notre cause. Qu'ils ne se gênent surtout pas ! (ALF 2011-02: 12)

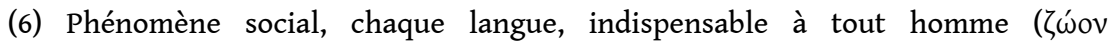

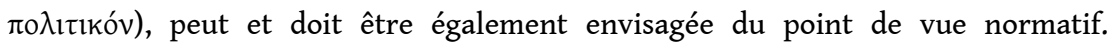
Appartenir à un domaine linguistique, c'est aussi le vouloir. Il n'est donc pas illégitime, contrairement à une tradition qui remonte, en France, à André Martinet, d'analyser les conséquences sur la langue du « vouloir vivre ensemble » dont parlait Renan, ainsi que la manière dont ce consensus peut être menacé par la désertion de l'« élite » de la communauté linguistique. Désertion, défaitisme - on pourrait dire pétainisme linguistique - d'ailleurs paradoxaux, car ceux qui prospèrent en affichant un déclinisme qui se conjugue comme un performatif pervers (" selffulfilling prophecy ») profitent du prestige d'une grande langue de culture, et devraient logiquement être les premiers à la défendre. (ALF 2011-02: 15)

(7) Je pense que le fait de tenter de remplacer "week-end" par "fin de semaine" est une utopie. On s'est tellement habitué a (sic!) cet anglicisme qu'il est difficile de distinguer le mot français du mot anglais. Si la volonté de se comporter le mieux possible n'empêche pas d'agir. Alors l'incapacité de réhabiliter certains mots et/ou expressions de français n'empêche pas de lutter contre l'anglicisme qui envahit le monde entier. (ABC/promotion linguistique 14.01.2011)

L'exemple (6) nous ramène finalement aux réflexions effectuées au début de cette contribution qui concernaient la difficulté de trouver des critères pertinents pour identifier des activités métalinguistiques populaires ou non-savantes. La discussion relative aux problèmes normatifs, liée au désir de certains acteurs métalinguistiques de 'purger' une langue, fait partie des deux genres discursifs, soit sous forme plutôt sousjacente (cf. (6)) soit parfois de manière stigmatisante en tant qu' 'exception française' (cf. (8) : " c'est une spécificité francophone »). Ce qui nous semble à la fois révélateur et paradoxal en ce qui concerne le discours puriste, c'est la valorisation de l'interventionnisme métalinguistique propre, par la délimitation consciente de l'autre (cf. (6)) d'une part, et par l'intégration du savoir de cet 'autre' dans le but se justifier soi-même (cf. (5)) d'autre part. Cela prouve que l'affection voire la passion portées à une langue dépend moins du fait qu'il s'agit d'un savoir scientifique ou populaire qui organise les représentations métalinguistiques (cf. (9)), que de l'idéologie représentée selon des critères spécifiques d'argumentation :

(8) Sur le purisme je suis d'accord, c'est une spécificité francophone et c'est parfois rigolo mais demander une certaine précision dans le langage n'est pas du purisme. (ABC/promotion linguistique 10.10.2011)

(9) Si le purisme manifeste un sentiment d'inconfort devant les autres façons de parler (il est donc normal que l'anglophobie soit sa version la plus répandue), il ne se donne pas nécessairement sous l'espèce de la maniaquerie ou de l'érudition philologique. ( $\mathrm{ABC} /$ promotion linguistique 01.08.2011) 


\section{Conclusion et perspectives}

30 L'étude des énoncés et attitudes métalinguistiques puristes ouvre de multiples perspectives et aujourd'hui c'est notamment l'approche de l'analyse du discours qui offre des modèles de recherche divers permettant aux linguistes de saisir des domaines intra- et transtextuelles tout en respectant en même temps le cadre sociohistorique d'un discours. Ainsi, en Allemagne, le domaine de recherche de la Diskurslinguistik propose un accès multifactoriel à la dimension 'transtextuelle' (Spitzmüller \& Warnke 2011), qui se situe théoriquement dans la proximité de la tradition foucaldienne ; sous cet angle un renforcement plus concret de l'alliance des théories et des méthodes de l'analyse du discours française et allemande nous semble avantageux.

31 La comparaison des genres discursifs a permis de montrer que les structures d'argumentation dans le discours puriste français sont construites selon les mêmes motifs dans différents lieux. De plus, les énoncés métalinguistiques laissent entrevoir que les attitudes puristes transmises idéologiquement par l'argumentation servent à construire et à établir un sentiment d'identité collective à la base de différents domaines topiques. En ce sens, la langue française est majoritairement discutée dans sa fonction sociale et non pas dans sa fonction communicative ou bien cognitive.

Étant donné que les acteurs métalinguistiques populaires représentent des participants importants dans l'ensemble du discours public sur la langue, les sciences du langage ont aussi la responsabilité de les intégrer davantage dans le cadre de recherches sociolinguistiques, ce qui leur permet en même temps de profiter des synergies qu'elles entretiennent avec d'autres disciplines. Au surplus, dans un tel cadre trans- et interdisciplinaire, des approches comparatives, dans des contextes nationaux divers, pourraient être utiles pour démontrer que la conscience métalinguistique puriste n'est pas une particularité française, mais qu'il s'agit plutôt d'un discours idéologique supranational.

\section{BIBLIOGRAPHIE}

$\mathrm{ABC}=\mathrm{ABC}$ de la langue française, sous : http://www.languefrancaise.net/ [dernière consultation 05.12.15].

$\mathrm{ABC} /$ promotion linguistique $=\mathrm{http}: / /$ www.languefrancaise.net $/$ forum/viewforum.php?id=2 [dernière consultation 05.12.15].

ALF 2015 = „Association de la langue française/Nos missions“, sous : http://www.avenir-languefrancaise.fr/articles.php?lng=fr\&pg=7\&mnuid=1164\&tconfig=0 [dernière consultation 12.11.15].

ALF 2011-01 = Avenir de la langue française. Journal de l'association Avenir de la langue française, 2011/1 (42), sous : http://www.avenir-langue-francaise.fr/file/journal/Bulletin42-mars2011.pdf [dernière consultation 13.11.15]. 
ALF 2011-02 = Avenir de la langue française. Journal de l'association Avenir de la langue française, 2011/1 (42), sous : http://www.avenir-langue-francaise.fr/file/journal/Bulletin43-juin2011.pdf [dernière consultation 13.11.15].

Authier-Revuz J. (2002). Le fait autonymique: langage, langue, discours - quelques repères. Sous $<$ http//www.cavi.univ-paris3.fr/ilpga/autonymie/actes.htm> [dernière consultation 03.12.2015].

Beacco J.-C. (2004a). Représentations métalinguistiques ordinaires et discours [= Langages 154]. Paris : Larousse.

Beacco J.-C. (2004b). Trois perspectives linguistiques sur la notion du genre discursif. Langages 38/153. 109-119.

Berrendonner A. (1982). L'éternel grammairien. Étude du discours normatif. Bern : Peter Lang. Bourdieu P. \& Boltanski L. (2008). La production de l'idéologie dominante. Éditions Demopolis. Cooper R. L. (1989). Language planning and Social Change. Cambridge.

Eckkrammer E. \& Lescure S. (2015). Aménagement linguistique et défense institutionnalisée de la langue : France. In Polzin-Haumann C. \&Schweickard, W. (eds). Manuel de linguistique française [= Manuals of Romance Linguistics, vol. 8]. Berlin/Boston : de Gruyter. 133-159.

Foucault M. (1969). L'archéologie du savoir. Paris : Gallimard.

Guespin L. \& Marcellesi J.-B. (1986). Pour la glottopolitique. Langages 83. 5-34.

Haugen E. (1959). Planning for a standard language in modern Norway. Anthopological linguistics 1/3. 8-21.

Julia C. (2001). Fixer le sens? La sémantique spontanée des gloses de spécification du sens. Paris : Presses Sorbonne Nouvelle.

Kienpointner M. (1983). Argumentationsanalyse. Innsbruck : Verlag des Instituts für Sprachwissenschaft.

Kopperschmidt M. (1989). Methodik der Argumentationsanalyse. Stuttgart/Bad-Canstatt : Frommann-Holzboog.

Labrie N. \& Nelde H.-P. (1994). L'aménagement linguistique dans la communauté européenne. In Carton F. \& Delefosse O. (eds). Les langues de l'Europe de demain. Paris : Presses Sorbonne Nouvelle. 117-127.

Maingueneau D. \& Cossutta F. (1995). L'analyse des discours constituants. Les analyses du discours en France (= Langages 117). 112-125.

Maingueneau D. (2007). Genres de discours et modes de généricité. Le français aujourd'hui 2007/4. 29-35.

Maingueneau D. (2014). Discours et analyse du discours. Paris : Armand Colin.

Niedzielski N. \& Preston D. (2000). Folk linguistics. New York : de Gruyter.

Osthus D. (2003). Le bon usage d'internet - discours et conscience normatifs dans des débats virtuels. In Osthus D., Polzin-Haumann C., Schmitt C. (eds). La norme linguistique. ThéoriesPratiques-Médias-Enseignement. Bonn : Romanistischer Verlag. 139-152.

Osthus D. (2015). Linguistique populaire et chroniques de langage : France. In Schweickard W.,Polzin-Haumann C. (eds). Manuel de linguistique française [= Manuals of Romance Linguistics, vol. 8]. Berlin/Boston : de Gruyter. 160-170. 
Ottmers C. (2007). Rhetorik. Stuttgart/Weimar : Metzler.

Paveau M.-A. (2007). Les normes perceptives de la linguistique populaire. Langage et société 119/1. 93-109.

Paveau M.-A. (2008). Les non-linguistes font-ils de la linguistique ? Pratiques 139/140. 93-109. URL : http://journals.openedition.org/pratiques/1200.

Paveau M.-A. \& Rosier L. (2008). La langue française : Passions et polémiques. Paris : Vuibert.

Paveau M.-A. \& Achard-Bayle G. (2008). Présentation. La linguistique « hors du temple ». Pratiques 139-140 : Linguistique populaire? sous : http://pratiques.revues.org/1171 (dernière consultation 17.09.15). 3-16.

Perelman C. \& Olbrechts-Tyteca L. (1970 (2004)). Die neue Rhetorik. Stuttgart/Bad Canstatt : Frommann-Holzboog.

Peyre H. (1933). La royauté et les langues provinciales. Paris : Presses modernes.

Polzin-Haumann C. (2008). Sprachplanung, Sprachlenkung und institutionalisierte Sprachpflege: Französisch und Okzitanisch. In Glessgen M.-D., Schmitt C. \& Schweickard W. (eds). Romanische Sprachgeschichte vol. 2. Berlin/New York : de Gruyter. 1472-1486.

Rey-Debove J. ([1978]/1997). Le métalangage. Étude linguistique du discours sur la langue. Paris : Armand Colin.

Rosier L. (2004). La circulation des discours à la lumière de l'effacement énonciatif : l'exemple du discours puriste sur la langue. Langages 38/156. 65-78.

Schmitt C. (2000). Nation und Sprache : Das Französische. In Gardt A. (éd.). Nation und Sprache vol. 2. Berlin/New York : de Gruyter. 673-745.

Spitzmüller J. (2005). Metasprachdiskurse. Einstellungen zu Anglizismen und ihre wissenschaftliche Rezeption. Berlin/New York : de Gruyter.

Spitzmüller J. \& Warnke I. H. (2011). Diskurslinguistik. Einführung in die Theorien und Methoden der transtextuellen Sprachanalyse. Berlin/Boston : de Gruyter.

Stegu M. (2008). Linguistique populaire, language awareness, linguistique appliquée : interrelations et transitions. Pratiques 139-140. 81-92.

Teulat R. (1975). Renaissantisme occitan e normalisacion. Quaserns de Lingüistica Occitana 2. 2-28.

Wengeler M. (2003).Topos und Diskurs. Begründung einer argumentationsanalytischen Methode und ihre Anwendung auf den Migrationsdiskurs (1960-1985). Tübingen : Niemeyer.

Wilton A. \& Stegu M. (2011). Bringing the 'folk' into applied linguistics. AILA Review 24. 1-14.

\section{NOTES}

1. Philippe de Commynes, historiographe au service de Louis XI, décrit que le roi réclamait « que en ce royaume l'on usat d'une coustume et d'ung poys et d'une mesure, et que toutes ces coustumes fussent mises en françoys en ung beau livre, pour éviter la cautelle et la pillerye des avocatz » (Peyre 1933: 12). Ce processus d'unification des règles juridiques au 15e siècle entraînait à sa suite le remplacement du latin et l'unification linguistique, entamée officiellement par l'Ordonnance de Villers-Cottêrets en 1539, l'acte décisif pour l'usage officiel du français dans les documents publics de justice et d'administration. 
2. Paveau \& Achard-Bayle (2008: 5) : «Le terme linguistique populaire est un calque d'une série de dénominations anglo-saxonnes basées sur folk, dans lesquelles folk est traduit en français par

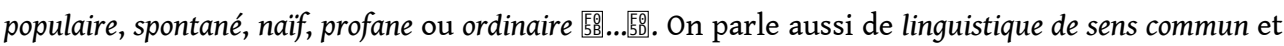
l'on rencontre également l'expression linguistique des profanes, dont L. Rosier signale la présence désormais massive sur l'internet: «On peut [...] ajouter ce qu'on nomme "la linguistique des profanes”, particulièrement visible sur l'internet, notamment dans le cadre des forums de discussion [...]» (Osthus 2003: 139, cité dans Rosier 2004: 70).

3. Sachons que si les puristes comme les non-savants ne peuvent pas être délimités à une certaine sphère sociale, ils peuvent néanmoins poursuivre les mêmes intérêts sociaux et agir, si l'on peut dire, comme groupe social à part.

4. Toute mise en relief par l'auteure.

\section{RÉSUMÉS}

Cet article se propose d'étudier le discours puriste sur la langue française dans une perspective comparative par l'opposition de deux genres d'interactionnisme discursif. Partant d'un aperçu sur la tradition métalinguistique en France et sur la notion d'aménagement linguistique, nous nous consacrons d'abord aux recherches liées au programme de la linguistique populaire. Dans ce contexte, notre contribution essaie tout d'abord de situer les acteurs du discours puriste et leurs actions verbales sur un axe qui oppose les constructions savantes aux constructions 'populaires'. Dans le but de définir les lieux communs idéologiques véhiculés dans les énoncés des acteurs puristes, s'exprimant dans des lieux et par des genres de communication différents, les approches de l'analyse de discours (AD) nous serviront de cadre méthodologique. Dans cette optique, l'analyse s'intéresse principalement aux topoï structurant le discours puriste, dans lequel savoirs et représentations métalinguistiques spécifiques et récurrents constituent un inventaire de réflexions partagées par les puristes.

This article considers the purist discourse of French language from a comparative perspective by analyzing the opposition of two discursive genres of metalinguistic interaction. Firstly, the article will be introduced by a discussion of the metalinguistic discourse tradition in France and the field of language planning. Secondly, the folk linguistic research and its interests will be illustrated. Considering the verbalized forms of cognitive representations in linguistic discourse, the focus will be on defining the actor's opinion of the continuum between an expert and a layperson. The ideologically constructed commonplaces conveyed by purist actors using different communication areas and genres lead to the introduction of discourse linguistics as an appropriate approach within the field of Applied Linguistics. Following this theoretical background, the objective of this paper is to reveal specific topical structures and argumentative layouts in order to gain new insights into the underlying beliefs of purism. 
INDEX

Mots-clés : métalangage - aménagement linguistique - linguistique populaire - analyse de discours - représentation

Keywords : metalanguage - language planning - folk linguistics - discourse analysis - cognitive representation

\section{AUTEUR}

VERA NEUSIUS

Université de la Sarre, Sarrebruck/Allemagne 